\title{
Molecular characterization of the $\mathrm{Na}^{+} / \mathrm{H}^{+}$-antiporter NhaA from Salmonella Typhimurium
}

Christopher J. Lentes ${ }^{1,2}$, Syed H. Mir ${ }^{1,3}$, Marc Boehm ${ }^{4}$, Constanta Ganea ${ }^{5}$, Klaus Fendler ${ }^{6}$, Carola Hunte ${ }^{1}$

${ }^{1}$ Institute for Biochemistry and Molecular Biology, ZBMZ, BIOSS Centre for Biological Signalling Studies, University of Freiburg, Freiburg, Germany; ${ }^{2}$ Faculty of Biology, University of Freiburg, Freiburg, Germany;

${ }^{3}$ Dept. Clinical Biochemistry, University of Kashmir, Srinagar, India; ${ }^{4}$ Dept. Molecular Membrane Biology, Max Planck Institute of Biophysics, Frankfurt/M., Germany; ${ }^{5}$ Biophysical Department, Faculty of Medicine, Carol Davila University of Medicine and Pharmacy, Bucharest, Romania; ${ }^{6}$ Dept. Biophysical Chemistry, Max Planck Institute of Biophysics, Frankfurt/M., Germany

Correspondence to Carola Hunte: Institute for Biochemistry and Molecular Biology, ZBMZ, BIOSS Centre for Biological Signalling Studies, Stefan-Meier-Strasse 17, University of Freiburg, 79104 Freiburg, Germany, E-mail:carola.hunte@biochemie.uni-freiburg.de

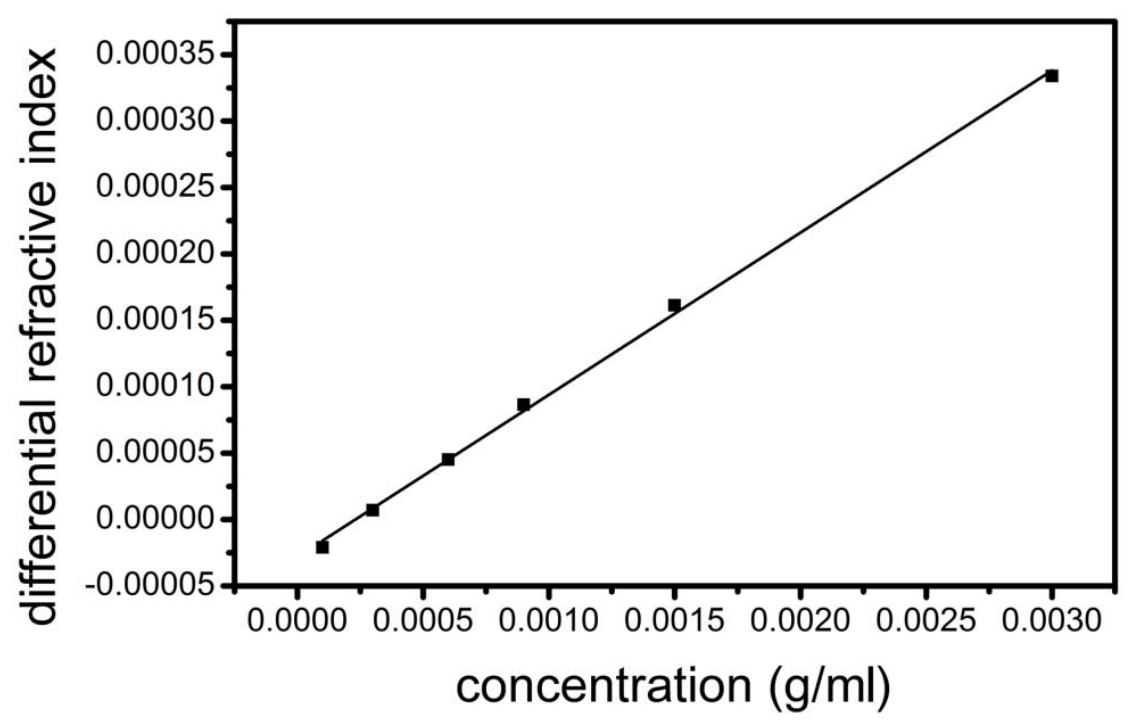

Figure S1. Determination of the $\mathrm{d} n / \mathrm{d} c$ value for $\beta$-DDM in SEC buffer. DDM concentration is given in $\mathrm{g} / \mathrm{ml}$. The $\mathrm{dn} / \mathrm{dc}$ for $ß-D D M$ was determined to be $0.122 \mathrm{~mL} / \mathrm{g}$. 